\title{
The redshifted footpoints of coronal loops
}

\author{
I. E. Dammasch ${ }^{1}$, W. Curdt ${ }^{2}$, B. N. Dwivedi ${ }^{2,3}$, and S. Parenti ${ }^{1}$ \\ ${ }^{1}$ Royal Observatory of Belgium, Brussels, Belgium \\ ${ }^{2}$ Max-Planck-Institut für Sonnensystemforschung, Katlenburg-Lindau, Germany \\ ${ }^{3}$ Department of Applied Physics, Banaras Hindu University, Varanasi, India
}

Received: 18 September 2007 - Revised: 22 November 2007 - Accepted: 28 November 2007 - Published: 15 October 2008

\begin{abstract}
The physics of coronal loops holds the key to understanding coronal heating and the flow of mass and energy in the region. However, the energy source, structure maintenance and mass balance in coronal loops are not yet fully understood. Observations of blue- and redshifted emissions have repeatedly been used in the construction of loop models. But observations and interpretations of line shifts have been widely debated. Here we present detailed SUMER observations, which clearly show a steady downflow in both footpoints of coronal loops observed at transition region (TR) and lower corona temperatures. We also show and quantify a correlation existing between this Doppler shift and the spectral radiance. Our results indicate a strong correlation which holds from the chromosphere to the lower corona. We suggest that the downflow in the footpoints may be a common phenomenon on all scales, which could explain, why on a statistical basis bright pixels tend to be more redshifted. We conclude by presenting interpretation of such results and their implications in the light of a viable coronal loop model. The observation of steady downflow in redshifted footpoints seems to be in conflict with impulsive heating.
\end{abstract}

Keywords. Solar physics, astrophysics, and astronomy (Corona and transition region) - Space plasma physics (Charged particle motion and acceleration; Transport processes)

\section{Introduction}

The redshift observed in emission lines seems to be an inherent property of the stellar transition region (e.g., KjeldsethMoe, 2003), indicating the presence of downflowing plasma. A mass flux at temperatures $T \approx 10^{5} \mathrm{~K}$ - sufficient to empty the corona in a few minutes - must rule out the possibility of

Correspondence to: I. E. Dammasch

(dammasch@oma.be) a true net downflow. Consequently, the apparent downflow may likely result from a spatial and/or temporal averaging of the plasma motion. The inference of temperature, density and velocity as a function of time and space is indispensable to develop any realistic model. At the same time, accurate deduced values are still missing. For instance, one may expect upflow to appear at temperatures different from transition region. However, there seems to be a compelling physical reason to believe that the motion of material becomes more visible while it is descending and it becomes less visible while ascending, at least with the observations available.

In order to understand the physics of the Sun's atmosphere from the redshifted and blueshifted emissions, a lot more effort is called for not only from high-resolution spatial and temporal observations but also theoretical modeling. A vast literature exists on the topic from the Skylab era to SOHO and TRACE (e.g., Doschek et al., 1976; Antiochos, 1984; McClymont, 1989; Hansteen, 1993; Brekke, 1993; Antiochos, 1994; Müller et al., 2004; McIntosh and Poland, 2004, and references therein).

\section{Downflows in AR loops and sunspot plumes}

In Figs. 1-3 we present three examples of active region (AR) loops, which exhibit redshifted emission at both legs.

The redshift at both loop legs is indicative of a steady and continuous downflow into the footpoint area at both polarities. In all Doppler maps presented here (Figs. 1, 2, 3, $5,6)$ the range of the flows spans from $-30 \mathrm{~km} / \mathrm{s}$ (blue) to $+30 \mathrm{~km} / \mathrm{s}$ (red).

Strong downflows are often observed in sunspot plumes (e.g., Brynildsen et al., 1998). These plumes can live for days and have always systematic downflows of up to $25 \mathrm{~km} / \mathrm{s}$. Often elongated redshifted features terminate in the plume area.

Published by Copernicus Publications on behalf of the European Geosciences Union. 

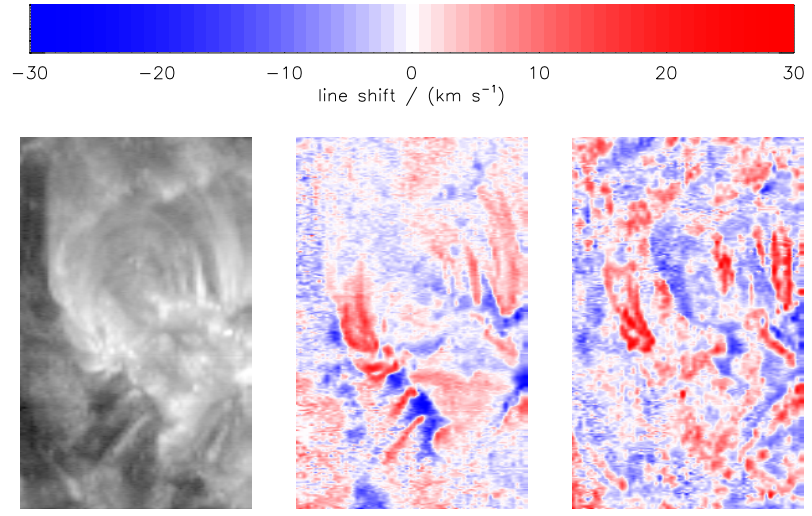

Fig. 1. Example of redshifted loop legs from 21 May 2004 showing Ne VIII emission both as radiance (left) and as Doppler map (center). It is also seen in the emission of O IV (right). The Doppler map reveals strong redshift of $20-30 \mathrm{~km} / \mathrm{s}$ at both loop legs. These observations suggest a quasi-continuous downflow at all TR temperatures. The FOV is $187^{\prime \prime} \times 300^{\prime \prime}$.
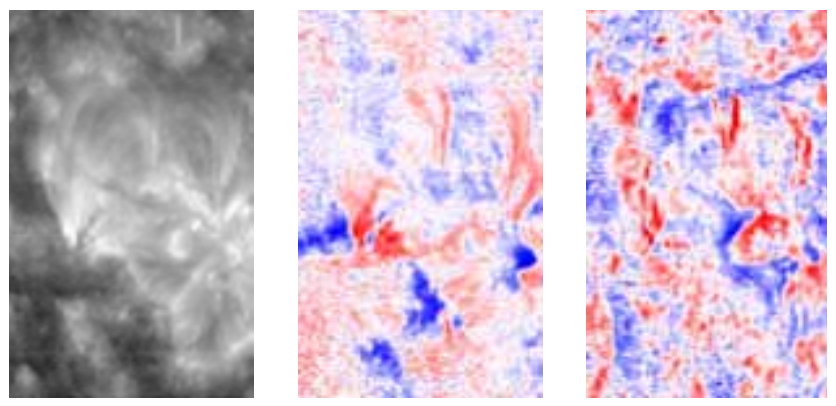

Fig. 2. Another example similar to Fig. 1, again showing Ne VIII radiance, Ne VIII Doppler shifts, and O IV Doppler shifts, obtained on 23 May 2004. The redshift in both legs seems to be a common feature of AR loops.

We infer that sunspot plumes are nothing else than the common footpoint of several active region loops, and that the processes are similar to those in normal active region loops. Recent results from a joint observation of the EIS spectrometer on Hinode and the MDI and SUMER instruments on SOHO corroborate this interpretation. Teriaca et al. (2007) deduce from a magnetic field extrapolation that many of the "radially directed shorter loops in the corolla around the sunspot" are at one end anchored in the plume.

\section{Downflows in the quiet Sun network}

It is well known, but not yet understood, that the average emission in transition region lines is redshifted by several $\mathrm{km} / \mathrm{s}$ (Brekke et al., 1997; Chae et al., 1998). This is also clearly visible (although not mentioned in the paper) from
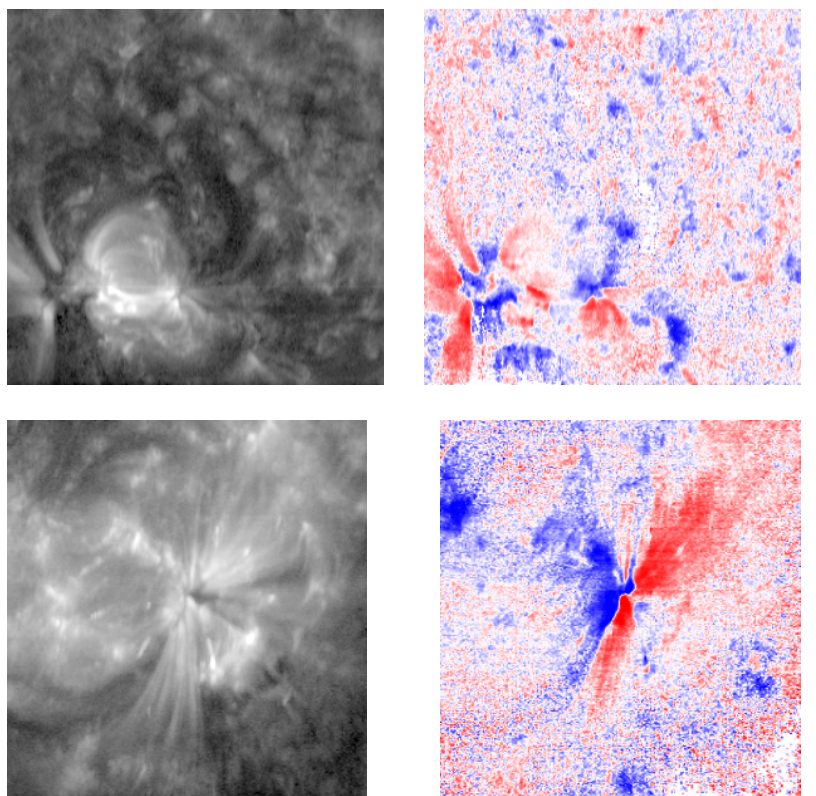

Fig. 3. Two examples of sunspot plumes from 23 March 1996 (top, FOV $=300^{\prime \prime} \times 300^{\prime \prime}$ ) and from 16 November 2006 (bottom, $\left.\mathrm{FOV}=287^{\prime \prime} \times 300^{\prime \prime}\right)$, shown as radiance map and as Doppler map. In both cases the sunspot was observed in Ne VIII.

the network contrast presented in the SUMER spectral atlas (Curdt et al., 2001). From the cutout of the original figure - shown here as Fig. 4 - it is seen that the network contrast increases by a factor of $>2$ in transition region lines and that the peaks are clearly redshifted. The observation that in the network, the transition region emission is enhanced and systematically redshifted, is well established. The new aspect introduced here is the assumption that in the quiet Sun (QS) a similar effect is at work as in the AR loops presented in Sect. 1. If we follow the suggestion of Feldman et al. (2001) and infer that the tiniest loops, too, are redshifted at both legs, we can explain the average redshift.

As suggested by Feldman et al. (2001) for chromospheric and TR loops, it is likely that unresolved fine-structured loops are anchored in the network. A statistical analysis was done to reveal more details.

\section{Redshift-brightness relationship}

There are several ways of describing the interrelation between brightness and redshift, e.g., (a) scatter plots, (b) scatter plots with a linear regression, (c) calculation of a correlation coefficient, (d) contour plots on 2-D images, and (e) clustered averages. While scatter plots are often difficult to interpret, regressions and correlation coefficients reduce the functional relationship of many data points to a single scalar. We have used techniques (d) and in particular (e) in this 


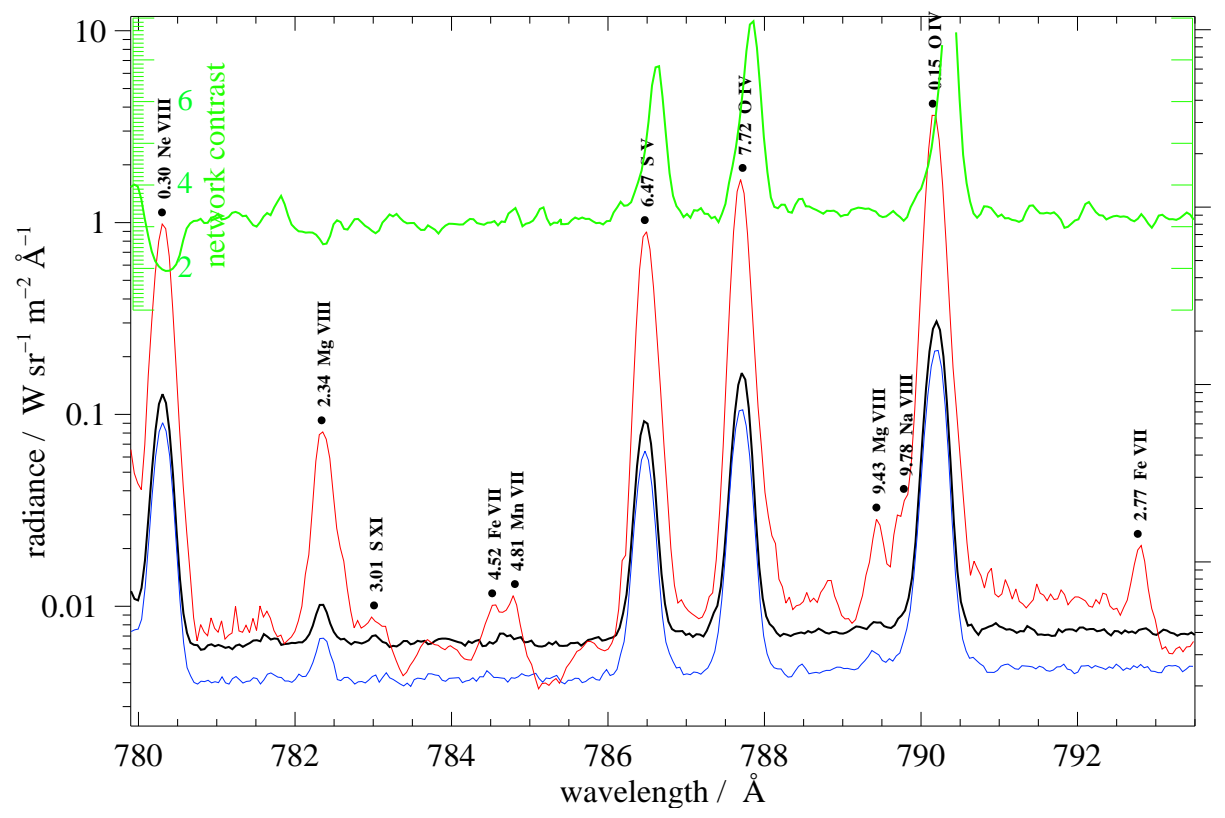

Fig. 4. Cutout of the SUMER spectral atlas (Curdt et al., 2001) showing radiances of average QS (black), sunspot (red), coronal hole (blue). The network contrast (ratio bright-network/cell-interior in green) increases by a factor of $>2$ in TR lines and the peaks are clearly redshifted.

paper, which exhibit the information contained in the data in an optimum way. Contour plots are restricted to only few levels, e.g., the $50 \%$ brightness quantile that we have used in Figs. 5 and 6.

By this method, a 2-D raster image of $m \times n$ pixels is converted into a vector of $m \cdot n$ elements, each with a radiance and a Doppler shift value. The vector is sorted by radiance and then segmented into clusters of 1000 elements. For each cluster, the average radiance and the average shift are calculated. Thus, we obtain a functional relationship between brightness and velocity, which is in many cases far from being linear. For instance, in the case of an AR within a larger QS region, the average shift can go from blue to neutral (QS pixels) and then back to blue and to red (in the brighter AR pixels). Single parameters, such as the correlation coefficient are not appropriate to express this kind of information, the reason being the fact that even if the coefficient states "no correlation", there is still a functional relationship, which may be meaningful.

Both the probability distributions of the logarithm of the QS radiances and Doppler shifts have a Gaussian shape (cf., Dammasch and Wilhelm, 2002). The lognormal distribution of these properties is quite different in chromospheric, TR and coronal emission. In QS emission, TR radiance intervals (i.e., the widths of their respective Gaussians) cover the widest range as well as shift intervals. The AR plots show, in what way the relations are similar in active regions, and in what way they are different.

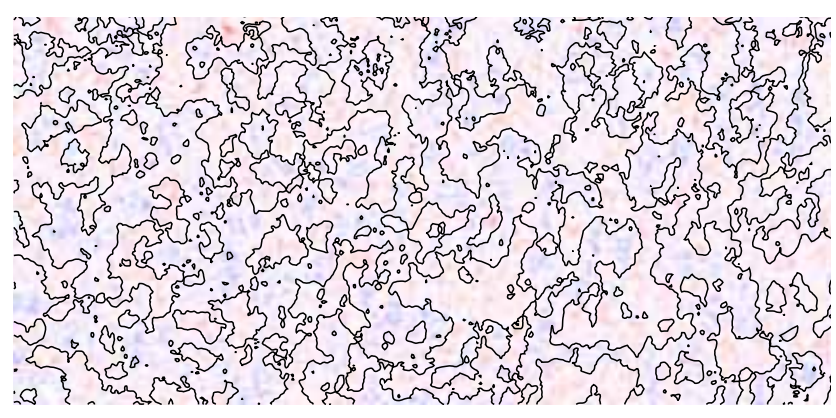

Fig. 5. QS raster in Si II 1533, obtained on 7 November 1999. The FOV is $633^{\prime \prime} \times 300^{\prime \prime}$. Brightness contours, which are overlaid on shifts nicely outline the redshift/blueshift boundary.

We have analysed a large quiet Sun raster scan obtained in the emission of upper chromospheric $\mathrm{Si}$ II $(\log T=4.13)$ at $1533 \AA$ to demonstrate quantitatively, that there is indeed a relationship between redshift and pixel brightness. In Fig. 5 we display the Doppler shifts found in this raster, overlaid by brightness contours. It is obvious that the contours outline the redshift/blueshift boundary. There is a clear trend of brighter pixels towards redshift.

In AR maps (cf., Fig. 6) a similar relationship is found in chromospheric and in TR lines. In coronal lines, however, an additional effect is observed. Here some small and dim, less blueshifted patches seem to result from upflows in confined structures. We have excluded explosive events from 

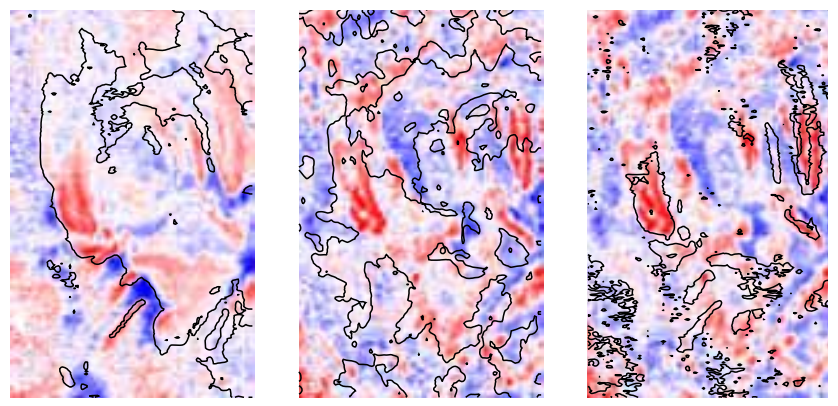

Fig. 6. A similar relationship is observed in the active region loops already shown in Fig. 1: magnetically confined structures are outlined by brightness contours within Ne VIII shifts (left) and within O IV shifts (center); structures are also outlined by Ne VIII redshift contours within O IV shifts (right).

this analysis, since they are short-lived transient phenomena, which behave differently and could possibly confuse our statistical results on the quasi-stationary AR loops.

Our statistical results are presented in Fig. 7, where we show redshift-versus-radiance diagrams for a quantitative description of the effect from the QS study of November 1999 in Si II 1533 (upper left), C IV 1548 (upper right, $\log T=5.1$ ), and Ne VIII 770 (lower left, $\log T=5.8$ ). In Fig. 7 we also show for comparison the Ne VIII plot (lower right) from an AR study in May 2004. Pixels are sorted according to brightness, 1000 pixels averaged. The interrelation between brightness and redshift holds for the chromosphere, for the TR (except in explosive events), and for the lower corona. Here, one has to differentiate between QS (partial relation) and AR (strong relation). This analysis expands the work reported by Brynildsen et al. (1998) and Doschek et al. (2004).

\section{Conclusions}

Our observations clearly show the signature of the downflow at both footpoints of quiescent AR loops in all TR emission lines observed by the SUMER spectrograph. We now infer that this phenomenon occurs in all the magnetically confined structures which is in agreement with the interpretation of sunspot plumes being the common footpoints of many loops. We also infer that the same may be valid for unresolved fine structures (UFS), already noted by Feldman et al. (2001). If we assume that the bright network consists of UFSs, we may explain the average redshift in TR lines (Chae et al., 1998; Brekke et al., 1997), since - effect of the increased density - the footpoint pixels are inherently brighter. This finding is supported by statistical analyses of Brynildsen et al. (1998), Doschek et al. (2004), Doschek (2006), and by the bright network/cell interior ratio presented by Curdt et al. (2001).

In this scenario, one important aspect is missing: the replenishment of the continuously downflowing material
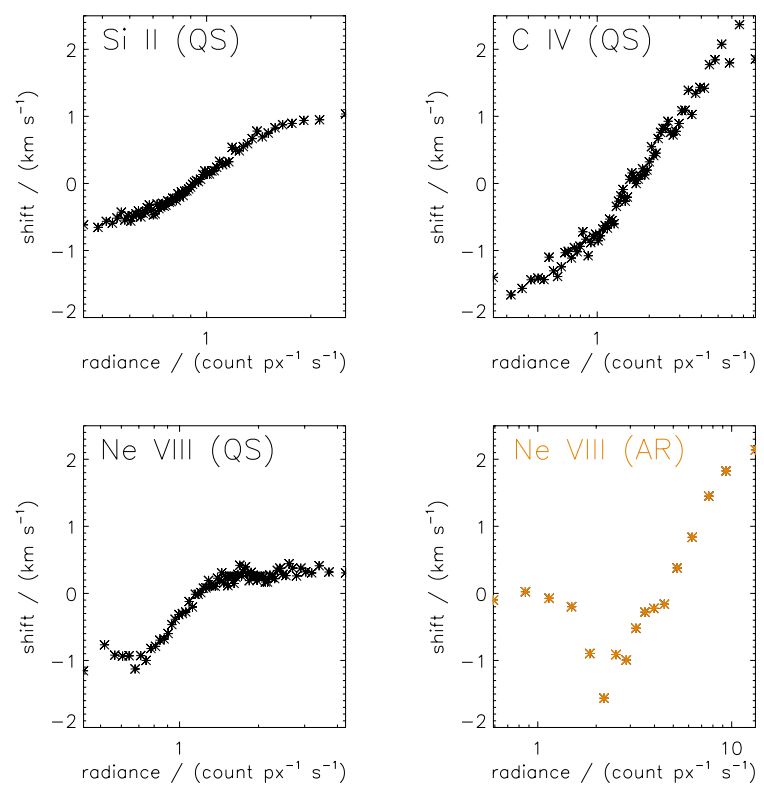

Fig. 7. The interrelation between brightness and Doppler shift is clearly seen in TR emission of the quiet Sun: Si II 1533, C IV 1548 , and Ne VIII 770. For comparison we also show a similar plot in $\mathrm{Ne}$ VIII for an AR (upper left to lower right).

into the magnetically confined structure. Different concepts have been discussed, including nanoflare generated waves (Hansteen et al., 1997), dark mottles (Tsiropoula and Tziotziou, 2004), or spicular evaporation (Budnik et al., 1998). Many models predict heating in loops to be impulsive (Klimchuk, 2006), assuming nano-flare generated chromospheric evaporation followed by condensation. Our observations do not allow to draw conclusions in favour of any of these models and it is beyond the scope of this communication to discuss the material balance problem. We can, however, say that redshift in loops which can stay unchanged for days do not support impulsive models and hint more at a steady and continous process. Possibly, the subdivision of loops into sub-resolution strands may provide an answer to this outstanding question. Observations at a higher resolution may be needed to allow progress in this field.

Acknowledgements. The SUMER project is financially supported by DLR, CNES, NASA and the ESA PRODEX Programme (Swiss contribution). SUMER is part of SOHO, the Solar and Heliospheric Observatory, of ESA and NASA. The work of BND was supported by a grant of the Max-Planck-Institute for Solar System Research. Funding of IED and SP by the Belgian Federal Science Policy Office (BELSPO) through the ESA/PRODEX programme is hereby appreciatively acknowledged.

Topical Editor R. Forsyth thanks two anonymous referees for their help in evaluating this paper. 


\section{References}

Antiochos, S. K.: A dynamic model for the solar transition region, Astrophys. J., 280, 416-422, 1984.

Antiochos, S. K.: Mass flows in coronal loops, Space Sci. Rev., 70, 143-148, 1994.

Brekke, P.: Observed redshifts in $\mathrm{O} \mathrm{V}$ and downflows in the solar transition region, AAstrophys. J., 408, 735-743, 1993.

Brekke, P., Hassler, D. M., and Wilhelm, K.: Doppler Shifts in the Quiet-Sun Transition Region and Corona Observed with SUMER on SOHO, Sol. Phys., 175, 349-374, 1997.

Brynildsen, N., Brekke, P., Fredvik, T., Haugan, S. V. H., KjeldsethMoe, O., Maltby, P., Harrison, R. A., and Wilhelm, K.: SOHO Observations of the Connection Between Line Profile Parameters in Active and Quiet Regions and the Net Red Shift in EUV Emission Lines, Sol. Phys., 181, 23-50, 1998.

Budnik, F., Schröder, K.-P., Wilhelm, K., and Glassmeier, K.-H.: Observational evidence for coronal mass injection by "evaporation" of spicular plasma, Astron. Astrophys., 334, L77-L80, 1998.

Chae, J., Yun, H. S., and Poland, A. I.,: Temperature Dependence of Ultraviolet Line Average Doppler Shifts in the Quiet Sun, Astrophys. J. Suppl. S., 114, 151-164, 1998.

Curdt, W., Brekke, P., Feldman, U., Wilhelm, K., Dwivedi, B. N., Schühle, U., and Lemaire, P., Astron. Astrophys., 375, 591, 2001.

Dammasch, I. E., and Wilhelm, K.: SUMER observations of solar transition region structures and dynamics, Adv. Space Res., 30, 495-500, 2002.

Doschek, G. A., Bohlin, J. D., and Feldman, U.: Doppler wavelength shifts of transition zone lines measured in SKYLAB solar spectra, Astrophys. J., 205, L177-L180, 1976.

Doschek, G. A., Mariska, J. T., and Akiyama, S.: The Relationship of the Chromosphere to the Lower Solar Transition Region, Astrophys. J., 609, 1153-1163, 2004.
Doschek, G. A.: Doppler Shift Correlations in the Solar Transition Region, Space Sci. Rev., 649, 515-528, 2006.

Feldman, U., Dammasch, I. E., and Wilhelm, K.: On the Unresolved Fine Structures of the Solar Upper Atmosphere. IV. The Interface with the Chromosphere, Space Sci. Rev., 558, 423-427, 2001.

Hansteen, V.: A new interpretation of the redshift observed in optically thin transition region lines, Astrophys. J., 402, 741-755, 1993.

Hansteen, V., Maltby, P., Malagoli, A.: Are the Redshifts Observed in Transition Region Lines Caused by Magnetic Reconnection?, in: Magnetic Reconnection in the Solar Atmosphere, edited by: Bentley, R. D. and Mariska, J. T., ASP conf. series, 111, 116121, 1997.

Kjeldseth-Moe, O.,: The solar transition region, in: Dynamic Sun, edited by: Dwivedi, B. N., Cambridge University Press, 196216, 2003.

Klimchuk, J. A.: On Solving the Coronal Heating Problem, Sol. Phys., 234, 41-77, 2006.

McClymont, A. N.: Can cool loop flows explain "transition region" redshifts?, Astrophys. J., 347, L47-50, 1989.

McIntosh, S. W. and Poland, A. I.: Detailed SUMER Observations of Coronal Loop Footpoint Dynamics, Astrophys. J., 604, 449454, 2004.

Müller, D. A. N., Peter, H., and Hansteen, V. H.: Dynamics of solar coronal loops. II. Catastrophic cooling and high-speed downflows, Astrophys. J. , 424, 289-300, 2004.

Teriaca, L., Wiegelmann, T., Lagg, A., Solanki, S. K., Curdt, W., and Sekii, T.: Loop morphology and flows and their relation with the magnetic field, in S. Matthews, J. Davis, \& K. Shibata (eds.), Announcing first results from Hinode, Dublin 20-24 Aug 2007, ASP conf. series, in press, 2007.

Tsiropoula, G. and Tziotziou, K.: The role of chromospheric mottles in the mass balance and heating of the solar atmosphere, Astrophys. J. , 424, 279-288, 2004. 